\title{
Comparison of microdebrider-assisted endoscopic sinus surgery and conventional endoscopic sinus surgery for chronic rhinosinusitis with nasal polyps
}

\author{
Ramiya Ramachandran Kaipuzha', Nirmal Coumare Venkataramanujam², Padmanabhan \\ Karthikeyan², Davis Thomas Pulimoottil ${ }^{3}$ \\ ${ }^{1}$ Department of Otorhinolaryngology and Head and Neck Surgery, Jahra Hospital, Al Jahra, Kuwait \\ ${ }^{2}$ Department of Otorhinolaryngology, Mahatma Gandhi Medical College and Research Institute, Pondicherry, India \\ ${ }^{3} \mathrm{Al}$ Azhar Medical College and Super Specialty Hospital, Ezhalloor, Thodupuzha, Kerala, India
}

\begin{abstract}
OBJECTIVE. To study and compare the benefits of microdebrider-assisted endoscopic sinus surgery and conventional endoscopic sinus surgery in terms of subjective and objective improvement in symptoms of nasal polyposis.

MATERIAL AND METHODS. This study involved 60 patients with bilateral sinonasal polyposis scheduled to undergo Endoscopic Sinus Surgery. The patients were randomized into two groups: Group A - Conventional endoscopic sinus surgery and Group B - Microdebrider-assisted endoscopic sinus surgery.

RESULTS. There was a significant difference in the mean VAS at 3 months postoperatively in Group B, but no significant difference at 6 months postoperatively following either of the two methods. The mean time for surgery $(p<0.01)$ and the mean intraoperative blood loss $(\mathrm{p}<0.01)$ were significantly lower in Group B.
\end{abstract}

CONCLUSION. A well-trained surgeon with proper anatomical knowledge, good instruments, hypotensive anaesthesia, minimal mucosal injury and regular proper follow-up will have similar postoperative results with both methods.

KEYWORDS: nasal polyps, microdebrider, surgical blood loss, operative time.

\section{INTRODUCTION}

Nasal polyposis forms a unique component of an inflammatory response affecting the nasal and paranasal sinus mucosa. It usually affects $1-4 \%$ of the population and is seen quite frequently in practice. Surgery is required to attain satisfactory ventilation and drainage of the impaired sinuses by means of utilizing either the microdebrider or the conventional instruments for functional endoscopic sinus surgery (FESS), when medical management fails to resolve the condition ${ }^{1,2}$. FESS is most appropriately defined as a minimally invasive procedure with the help of an endoscope to re-establish nasociliary clearance of mucous, drainage and aeration of the sinuses ${ }^{3,4}$. Certainly, the introduction of the rigid endoscope for the diagnosis and management of sinonasal disorders has been the most revolutionary advancement in the field of rhinology ${ }^{5,6}$. Mucosal preservation is indispensable for attaining drainage of the sinuses. When the mucosa is removed, the surgeon should endeavour to reline the mucosal surface of the sinus. Rapid postoperative resumption of the mucociliary function is contingent on ciliary regeneration and reducing the amount of bone exposed via the surgical procedure due to radical excision of the mucosa ${ }^{7,8}$.

Powered sinus instruments were introduced into practice in FESS some time ago, with the development of the microdebrider. Microdebrider-assisted FESS is precise, with a relatively bloodless field, although the meticulousness of surgery is dependent

Corresponding author: Dr. Davis Thomas Pulimoottil, Address: Department of Otorhinolaryngology and Head and Neck Surgery, Al Azhar Medical College and Super Specialty Hospital, Ezhalloor, Thodupuzha, Idukki District, Kerala, India - 685608 
on the surgeon's precise knowledge of anatomy and operative skills, as there is a high risk for major complications with the imprecise or erroneous use of the microdebrider in FESS $^{9}$.

This study aimed to compare microdebrider-assisted endoscopic sinus surgery and conventional endoscopic sinus surgery for the treatment of patients with chronic rhinosinusitis with nasal polyposis in the Department of Otorhinolaryngology of a rural tertiary care centre, over a period of 2 years, in terms of the postoperative benefits and incidence of complications. There are no similar studies from this part of India in the literature and, owing to the growing widespread use of microdebriders in ENT practice, this study assumes importance.

\section{MATERIAL AND METHODS}

Following the approval of this study by the Institutional Medical Ethics Committee, from January 2014 until December 2015, 60 patients aged between 18-70 years diagnosed with bilateral sinonasal polyposis not responding to medical treatment and requiring Endoscopic Sinus Surgery were included. Written informed consent was obtained from the patients. The inclusion criteria were: all unoperated cases of bilateral nasal polyps presenting with nose block, nasal discharge and sneezing, combined modified Lund-Mackay score and visual analogue score (VAS) of greater than or equal to 20, CT scan of cases showing Lund-Mackay total score of equal to or more than 8 on each side and patients with bilateral nasal polyps who failed conservative therapy. The exclusion criteria were: patients with active infection, bleeding disorders, chronic granulomatous diseases of the nose and tumors of the nose, recurrent ethmoidal polyps with history of previous surgery; patients with unilateral nasal polyposis and patients with contraindications to general anaesthesia.

All patients underwent medical treatment, involving a brief course of systemic steroids for two-week duration and a topical nasal steroid spray for a month, but with persistence of the disease. All patients underwent thorough medical history and clinical examination, which was promptly recorded on a medical sheet. The patients were then subjected to basic preoperative blood investigations, absolute eosinophil count, plain X-ray of the nose and paranasal sinuses (Water's view), Diagnostic Nasal Endoscopy (DNE) and plain CT of the nose and paranasal sinuses (coronal and axial views). These patients were randomized by the block randomization method into two groups consisting of 30 patients each: Group A - Conventional endoscopic sinus surgery and Group B - Microdebrider-assisted endoscopic sinus surgery. All patients completed a preoperative visual analogue scale (VAS) and a modified Lund-Mackay scoring to assess the severity and the impact of symptoms like olfactory disturbance, nasal discharge, facial pain, nasal blockage or congestion, headache and overall discomfort. The findings of DNE were scored based on the modified Lund-Mackay scoring system. Lund-Mackay scoring system for radiological grading was used to grade the disease on the CT scans.

After obtaining anaesthetic clearance for surgery, the patients were taken up for Endoscopic Sinus Surgery. Intraoperatively, the amount of blood loss (based on the collection of blood in the suction apparatus) and the duration of surgery (from the start of the surgery to nasal packing) were noted. In Group A, the Messerklinger technique was used with the help of conventional endoscopic sinus surgery instruments. In Group B, the microdebrider (Medtronic Xomed; Jacksonville, Fla.) was used for the surgical intervention. Postoperatively, the patients underwent saline nasal douches which were continued after discharge. The patients' follow-up was done up to the sixth month postoperatively to inspect for scarring, discharge, crusting, recurrence of polyps and reduction of symptoms based on the Lund-Mackay post-operative scoring system. Subjective evaluation of the patient was done using the visual analogue score (VAS).

The statistical analysis was done using SPSS version 19.0 software (IBM SPSS, US) with Regression Modules installed. A statistical comparison between the two groups was performed using Chi-square analysis for categorical variables and Student's t-test for continuous variables. All tests were two-tailed and the significance level was set at $\mathrm{p}<0.05$.

\section{RESULTS}

60 patients with bilateral sinonasal polyposis were included in the study. Overall, the mean age was $36.21 \pm 12.37$ years, with the maximum number of patients in the $2^{\text {nd }}$ and $3^{\text {rd }}$ decades of life. A clear male preponderance $(56.67 \%)$ was noted (male:female ratio $=1.3: 1)$. The most common symptoms noted were nasal obstruction $(100 \%)$ and sneezing $(85 \%)$. The majority of patients $(76.67 \%)$ were found to have stage 2 diseases on endoscopic examination, which refers to polyps extending to areas beyond the middle conchae without reaching the floor of the nasal passage. None of these findings were found to be statistically significant.

The preoperative overall mean VAS for symptoms in Group A was $8.03 \pm 0.66$ and $8 \pm 0.65$ in Group B. 


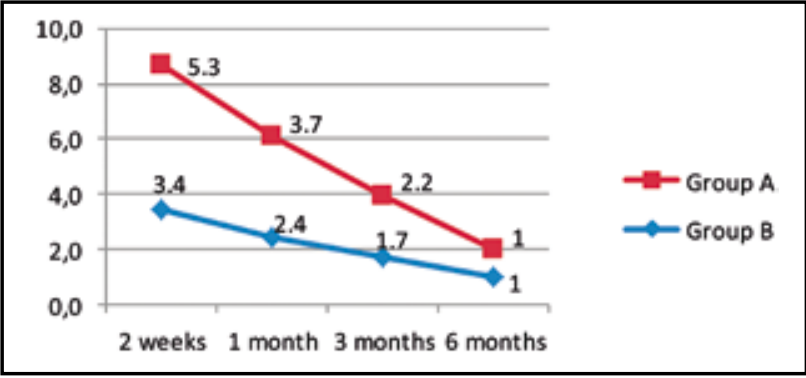

Figure 1 Comparison of mean VAS scores for nasal obstruction.

Figures 1-3 show the comparison of the mean VAS for nasal obstruction, nasal discharge and overall mean VAS for symptoms. As it can be clearly seen, the initial VAS is lower for Group B, but the scores are almost similar for both groups 6 months after surgery. The comparison of postoperative VAS 3 and 6 months after surgery does not show any statistical significance, implying that short-term benefits were experienced in Group B (Table 1).

The mean estimated blood loss (EBL) was found to be lower $(126.33 \pm 25.36 \mathrm{~mL})$ in Group B, and the overall reduction of blood loss as compared to Group A was $12.19 \%$. The mean operative time was also found to be lower for Group B $(100.67 \pm 27.38$ $\mathrm{min}$ ), and the overall percentage reduction in mean operative time as compared to Group A was found to be $46.54 \%$. These results were found to be statistically very significant $(p=0.004125)$ (Table 2$)$.

Postoperative endoscopic examination of patients in both groups at 2 weeks, 1 month, 3 months

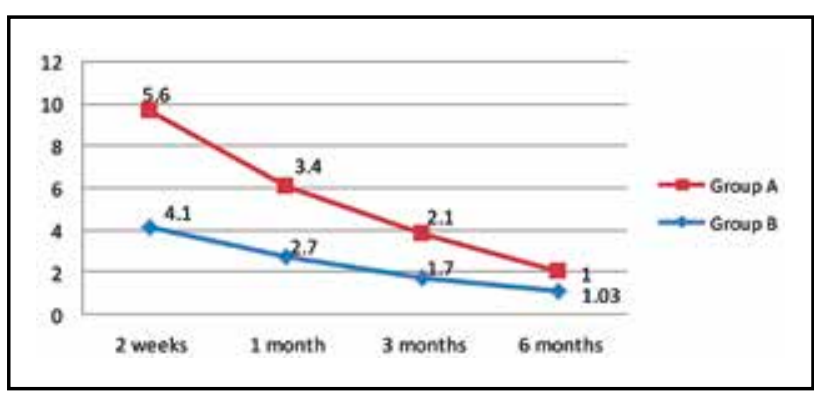

Figure 2 Comparison of Mean VAS scores for nasal discharge.

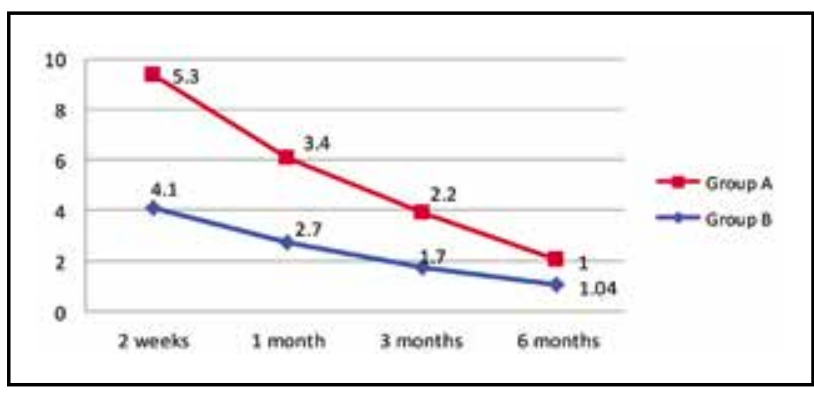

Figure 3 Comparison of overall Mean VAS scores for symptoms.

and 6 months revealed a much lower prevalence of crusting, synechiae and oedema in Group B patients as compared to Group A, the difference being statistically very significant $(p<0.01)$ (Table $3)$. Recurrence rates at the 6-month follow-up were found to be slightly higher in Group A $(6.67 \%)$ as compared to Group B (5\%), but this difference was not found to be statistically significant.

Table 1

Comparison between VAS scores 3 months and 6 months after surgery.

\begin{tabular}{|c|c|c|c|c|c|}
\hline VAS & GROUP & MEAN & STD DEVIATION & STD ERROR MEAN & $p$ VALUE \\
\hline \multirow{2}{*}{ VAS AT 3 MONTHS } & GROUP A & 2.17 & 0.06 & 1.25 & \multirow{4}{*}{0.873438} \\
\hline & GROUP B & 1.87 & 0.29 & 1.08 & \\
\hline \multirow[t]{2}{*}{ VAS AT 6 MONTHS } & GROUP A & 1.16 & 0.21 & 0.67 & \\
\hline & GROUP B & 1.03 & 0.06 & 0.59 & \\
\hline
\end{tabular}

Table 2

Comparison between type of surgery, estimated blood loss and operative time.

\begin{tabular}{ccccc}
\hline GROUP & $\begin{array}{c}\text { MEAN EBL } \\
(\mathbf{m L})\end{array}$ & $\begin{array}{c}\text { MEAN OPERATIVE TIME } \\
(\mathbf{O T})(\mathbf{m i n})\end{array}$ & $\begin{array}{c}\text { MEAN EBL / OT } \\
(\mathbf{m l} / \mathbf{m i n})\end{array}$ & $p$ VALUE \\
\cline { 1 - 3 } GROUP A & $143.87 \pm 20.39$ & $188.33 \pm 27.49$ & $1.32 \pm 0.35$ & 0.004125 \\
\cline { 1 - 4 } GROUP B & $126.33 \pm 25.36$ & $100.67 \pm 27.38$ & $0.78 \pm 0.16$ & \\
\hline
\end{tabular}


Table 3

Distribution of postoperative examination findings.

\begin{tabular}{|c|c|c|c|c|c|c|c|c|c|}
\hline \multirow[b]{2}{*}{ Findings } & \multicolumn{4}{|c|}{ GROUPA } & \multicolumn{4}{|c|}{ GROUP B } & \multirow[b]{2}{*}{$p$ VALUE } \\
\hline & $\begin{array}{c}2 \text { weeks } \\
(\%)\end{array}$ & $\begin{array}{c}1 \text { month } \\
(\%)\end{array}$ & $\begin{array}{c}3 \text { months } \\
(\%)\end{array}$ & $\begin{array}{c}6 \text { months } \\
(\%)\end{array}$ & $\begin{array}{c}2 \text { weeks } \\
(\%)\end{array}$ & $\begin{array}{c}1 \text { month } \\
(\%)\end{array}$ & $\begin{array}{c}3 \text { months } \\
(\%)\end{array}$ & $\begin{array}{c}6 \text { months } \\
(\%)\end{array}$ & \\
\hline Crusting & $30(50 \%)$ & $29(48.33 \%)$ & $27(45 \%)$ & $0(0 \%)$ & $25(41.67 \%)$ & $10(16.67 \%)$ & $2(3.33 \%)$ & $0(0 \%)$ & 0.000932 \\
\hline Synechiae & $30(50 \%)$ & $30(50 \%)$ & $23(36.67 \%)$ & $0(0 \%)$ & $12(20 \%)$ & $1(1.67 \%)$ & $0(0 \%)$ & $0(0 \%)$ & 0.000702 \\
\hline Oedema & $30(50 \%)$ & $29(48.33 \%)$ & $19(31.67 \%)$ & $3(5 \%)$ & $23(38.33 \%)$ & $11(18.33 \%)$ & $1(1.67 \%)$ & $0(0 \%)$ & 0.00793 \\
\hline
\end{tabular}

\section{DISCUSSIONS}

According to the epidemiological analysis in patients with nasal polyps performed by Bettega $\mathrm{S}$ et al., polyps are more common in the elderly over the age of 50 and rarely affect children and young people ${ }^{10}$. This stands in contrast to the findings of this study and may be explained by a probable greater allergic predisposition of the population under study or the smaller sample size. The overall male to female ratio was found to be $1.3: 1$ in the present study. This agrees with other epidemiological studies which found a male preponderance in cases of nasal polyposis ${ }^{11}$.

In a study by Saafan et al. comparing powered instruments in FESS with conventional methods, both groups experienced a significant improvement in the VAS postoperatively. There was no statistically significant difference found between the powered endoscopic and the conventional instruments groups in the total or the individual VAS scores except for olfaction ${ }^{12}$. This finding was mirrored by Ceylan et al. in their comparative randomised single-blinded clinical study on the impact of the microdebrider in the surgical treatment of nasal polyps. The comparison of the preoperative and postoperative scores by the paired t-test showed a statistically significant difference $(p<0.05)$ between the two groups, but the comparison of the postoperative values for each group by one way ANOVA test showed no statistically significant difference $(p>0.05)^{13}$. The present study showed no differences in postoperative VAS at the long-term follow-up, suggesting that microdebrider-assisted endoscopic polypectomy offers short-term benefits.

A regular menace of conventional instruments is their tendency to tear tissues and strip the mucosa, leading to increased bleeding with decreased visibility and increased frequency of complications and scarring. Microdebriders offer suction at the surgical site and tender the advantages of remov- ing polypoid tissue from the surgical site devoid of the need to take out the instrument, thus providing continuous removal of blood from the field. This proffers improved visualization, allows better precision and has the advantage of fewer interruptions during surgery. Krouse and Christmas ${ }^{14}$ found that an average of $19.5 \mathrm{~mL}$ blood was reported for cases operated on with powered instruments and an average of $44.5 \mathrm{~mL}$ in the cases operated with conventional instruments, showing that surgical bleeding was reduced by more than half with microdebriders. This finding was mirrored by other studies performed by R Singh et al. ${ }^{15}$ and Kumar and Sindwani ${ }^{16}$. Similar results were seen in the present study too.

This study found that the mean percentage reduction in operative duration between the two groups was found to be $46.65 \%$, which was very significant $(\mathrm{p}<0.01)$. This reduction in the mean operative duration in the microdebrider group may be explained by the extended time required to control haemorrhage in certain cases of the conventional group. The microdebrider presents a more optimal operative field due to its inherent suction of both blood and tissues. Shorter operative times with microdebriders were also seen in studies by Saafan et al. ${ }^{12}$, although studies by Selivanova et al. ${ }^{17}$ and R Singh et al. ${ }^{15}$ could not find any significant difference in operating times between the two groups.

The most common complication that occurs in FESS is the formation of synechiae $(6-27 \%)$. The development of synechiae is contingent upon the contact between two opposing denuded mucosal surfaces during the process of healing. The presence of synechiae may either be an incidental finding or may sometimes cause symptomatic obstruction of the sinus outflow tract, based on the extent and site of the synechiae. Therefore, supreme importance should be accorded to the maximal reduction of trauma to tissue and the preservation of normal mucosa, in order to pre- 
vent excessive scarring, and this is the advantage of the microdebrider. Studies by Setliff ${ }^{18}$ and Bernstein et al. ${ }^{8}$ showed reduced synechiae, minimal crust formation and rapid mucosal healing with the use of the microdebrider. These finding were reflected in those of the present study too and serve to show the superior results of the microdebrider in endoscopic sinus surgery.

\section{CONCLUSIONS}

Treatment of the nasal polyps has seen many advances in the recent past with the use of powered instrumentation. Zero to minimal mucosal injury in microdebrider-assisted polypectomy results in minimal intraoperative bleeding and no synechiae formation as compared to conventional polypectomy, and it is associated with reduced operative duration and better postoperative outcomes in terms of crusting, synechiae and oedema.

Thorough anatomical knowledge with good radiological workup, adequate hands on training and periodical patient follow-up reduce recurrence with both conventional and microdebrider-assisted endoscopic polypectomy.

Acknowledgement: The authors would like to thank Dr. G. Ezhumalai, Senior Statistician, Sri Balaji Vidyapeeth University, Pondicherry, for the immense help proffered for the statistical analysis for this study. The authors are also grateful to the authors of all those articles from where the literature for this article has been reviewed and discussed.

Conflict of interest: The authors have no conflict of interest.

Contribution of authors: All authors have equally contributed to this work.

Ethical standards: The authors assert that all procedures contributing to this work comply with the ethical standards of the institutional research committee and with the Helsinki Declaration of 1964, as revised in 2008. All patients gave their informed consent for their inclusion in the study. This study does not contain any studies with animals performed by any of the authors.

\section{REFERENCES}

1. Mygind N, Lund JV, Jones RJ. Nasal polyposis and surgical management of rhinosinusitis. In: Gleeson M, Browning GG, Burton JM, Clarke R, Hibbert J, Jones NS, et al. Scott and Brown's Otorhinolaryngology: Head and neck surgery. 7th edition, volume 2. Edward Arnold Publishers Ltd; 2008, p.1549-50,1552-6,1480-1.

2. Davidson TM, Murphy C, Mitchell M, Smith C, Light M. Management of chronic sinusitis in cystic fibrosis. Laryngoscope. 1995;105(4 Pt 1):354-8.

3. Bachert C, Hörmann K, Mosges R, Rasp G, Riechelmann H, Muller R, et al. An update on the diagnosis and treatment of sinusitis and nasal polyposis. Allergy. 2003;58(3):176-91.

4. Nores JM, Avan P, Bonfils P. Medical management of nasal polyposis: a study in a series of 152 consecutive patients. Rhinology. 2003;41 (2):97-102.

5. Marks SC. Endoscopic sinus surgery. In: Marks SC. Textbook on nasal and sinus surgery. W.B. Saunders Company; 2000, p.119,125-6,140-1.

6. Becker SS. Surgical management of polyps in the treatment of nasal airway obstruction. Otolaryngol Clin North Am. 2009;42(2):377-85, x. DOI: 10.1016/j.otc.2009.01.002.

7. Bruggers $\mathrm{S}$, Sindwani R. Innovations in microdebrider technology and design. Otolaryngol Clin North Am. 2009;42(5):781-7, viii. DOI: 10.1016/j.otc.2009.07.003.

8. Bernstein JM, Lebowitz RA, Jacobs JB. Initial report on postoperative healing after endoscopic sinus surgery with the microdebrider. Otolaryngol Head Neck Surg. 1998;118(6):800-3.

9. Stammberger H. Functional endoscopic sinus surgery: The Messerklinger Technique. Mosby Inc; 1991

10. Bettega S, Soccol AT, Koerner HN, Mocellin M. Epidemiological analisys in patients with nasal polyps. Int Arch Otorhinolaryngol. 2007;11(3):243-7.

11. Larsen K, Tos M. The estimated incidence of symptomatic nasal polyps. Acta Otolaryngol. 2002;122(2):179-82.

12. Saafan ME, Ragab SM, Albirmawy OA, Elsherif HS. Powered versus conventional endoscopic sinus surgery instruments in management of sinonasal polyposis. Eur Arch Otorhinolaryngol. 2013;270(1):149-55. DOI: 10.1007/s00405-012-1969-8. Epub 2012 Apr 10.

13. Ceylan K, Bayiz U, Kizilkaya Z, Lale O, Emir H, Unlu I, et al. Impact of microdebrider in surgical treatment of nasal polyposis in terms of health related quality of life and objective findings: A comparative randomized single blinded clinical study. KBB-Forum. 2007;6(2):60-5.

14. Krouse JH, Christmas DA Jr. Powered instrumentation in functional endoscopic sinus surgery. II: A comparative study. Ear Nose Throat J. $1996 ; 75(1): 42-4$.

15. Singh R, Hazarika P, Nayak DR, Balakrishnan R, Gangwar N, Hazarika M. A comparison of microdebrider assisted endoscopic sinus surgery and conventional endoscopic sinus surgery for nasal polypi. Indian J Otolaryngol Head Neck Surg. 2013;65(3):193-6. DOI: 10.1007/s12070-0110332-5. Epub 2011 Nov 30.

16. Kumar N, Sindwani R. Bipolar microdebrider reduces intraoperative blood loss and operating time during nasal polyp surgery. Laryngoscope. 2009;119(S1):S143. DOI: 10.1002/lary.20459.

17. Selivanova O, Kuehnemund M, Mann WJ, Amedee RG. Comparison of conventional instruments and mechanical debriders for surgery of patients with chronic sinusitis. Am J Rhinol. 2003;17(4):197-202.

18. Setliff RC 3rd. The hummer: a remedy for apprehension in functional endoscopic sinus surgery. Otolaryngol Clin North Am. 1996;29(1):95-104. 